\title{
Handoff Rate Analysis in Heterogeneous Wireless Networks with Poisson and Poisson Cluster Patterns
}

\author{
Wei Bao and Ben Liang \\ Department of Electrical and Computer Engineering, University of Toronto \\ Toronto, Ontario, Canada \\ wbao@comm.utoronto.ca, liang@comm.utoronto.ca
}

\begin{abstract}
In multi-tier heterogeneous wireless networks (HWNs), both horizontal and vertical handoffs impact the signaling overhead and quality of service in the system. However, they are difficult to analyze due to the diverse and irregularly shaped cells in HWNs. The causes of this irregularity are three-fold: (1) small-cell base stations (BSs) tend to be deployed with a high level of spatial randomness; (2) BSs are likely to aggregate around highly populated geographical regions; (3) various transmission power levels in different tiers further create diverse cell sizes and shapes. In this work we present a new stochastic geometric analysis framework on user mobility in HWNs. Each tier of BSs is modeled as either a Poisson point process (PPP) or a Poisson cluster process (PCP), to capture their spatial randomness and their nonuniform and dependent aggregation in space. Flexible user association is also taken into consideration, such that various scales of cell sizes are accommodated. We derive analytical expressions for the rates of all handoff types experienced by an active user with arbitrary movement trajectory. We also demonstrate an example application of the proposed analysis, in optimizing the multi-tier BS selection by users, to balance the tradeoff between data rate and handoff overhead. Finally, extensive simulation is conducted to validate the correctness and usefulness of our analysis.
\end{abstract}

\section{Categories and Subject Descriptors}

C.2.1 [Network Architecture and Design]: Wireless communication

\section{Keywords}

Mobility; handoff; stochastic geometry; analytic geometry; Poisson cluster process

\section{INTRODUCTION}

Traditional single-tier macro-cellular networks provide wide coverage for mobile user equipments (UEs), but they are

Permission to make digital or hard copies of all or part of this work for personal or classroom use is granted without fee provided that copies are not made or distributed for profit or commercial advantage and that copies bear this notice and the full citation on the first page. Copyrights for components of this work owned by others than ACM must be honored. Abstracting with credit is permitted. To copy otherwise, or republish, to post on servers or to redistribute to lists, requires prior specific permission and/or a fee. Request permissions from permissions@ acm.org. MobiHoc'15, June 22-25, 2015, Hangzhou, China.

Copyright (C) 2015 ACM 978-1-4503-3489-1/15/06 ...\$15.00.

http://dx.doi.org/10.1145/2746285.2746317. insufficient to satisfy the exploding demand for high bandwidth access driven by modern mobile traffic. One effective means to increase network capacity is to provide more serving stations within a geographical area, i.e., installing a diverse set of small-cells such as femtocells and WiFi hotspots, overlaying the macrocells, to form a multi-tier heterogeneous wireless network (HWN). Each small-cell is equipped with a shorter-range and lower-cost base station (BS) or access point (AP), to give nearby UEs higher-bandwidth network access with lower power usage, and to offload data traffic from macrocells.

In the presence of multiple tiers of cells, however, mobility management of the UEs becomes more challenging. In particular, HWNs introduce vertical handoffs, i.e., handoffs made between two BSs in different tiers [30]. Compared with horizontal handoffs, i.e., handoffs made between two BSs in the same tier, vertical handoffs impact both the UEs and the overall system in more complicated ways. For example, extra traffic latency and additional network signaling are incurred during channel setup and tear down when a vertical handoff is made; more UE power may be consumed due to simultaneously active network interface to multiple tiers; call drops or degraded quality of service (QoS) could also be experienced by UEs due to the lack of radio resource after handoff.

We define the handoff rate as the expected number of handoffs experienced by one UE per unit time. It is closely related to the signaling overhead in the system and the QoS of UEs. As a prerequisite to performance evaluation and system design in HWNs, it is essential to quantify the rates of different handoff types. However, the analysis on handoff rates in HWNs is complicated by the irregularly shaped multi-tier network topologies introduced by the small-cell structure. First, small-cell BSs are often installed incrementally and irregularly, with a high level of spatial randomness. Second, BSs are likely to aggregate around some highly populated geographical regions (e.g., urban areas, attractions, etc.), which further complicates the spatial pattern of the network. Third, different tiers of BSs communicate at different power levels, causing various scales of cell sizes, and non-polygon cell shapes. As a consequence, it is difficult to characterize the cell boundaries and to track boundary crossings made by UEs (i.e., handoffs) in the system. Fig. 1(a) shows an example topology with two tiers of BSs. Previously developed analytical techniques in the literature, using queueing theory $[3,5,10,13,15,21]$, regular grids $[2,17,28]$, or homogeneous Poisson point processes (PPPs) [6, 9, 23], 


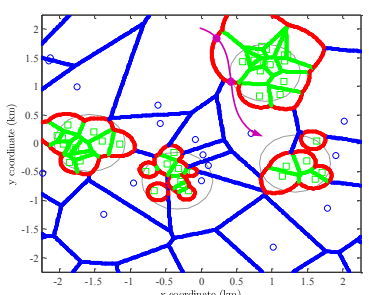

(a) The UE experiences two vertical handoffs.

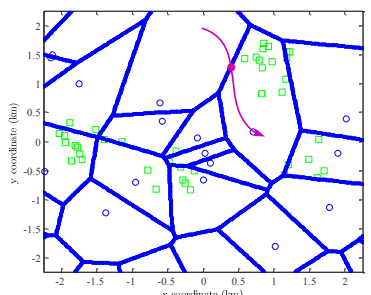

(b) Same UE trajectory with one horizontal handoff.

Figure 1: An example of a two-tier HWN. Tier-1 and 2 BSs are represented by " $\circ$ " and " $\square$ " respectively. Tier-2 BSs are clustered in four disk regions. Blue, red, and green curves show cell boundaries within tier-1, between tier-1 and tier-2, and within tier2 , respectively. The magenta arrow represents the trajectory of an active UE.

are insufficient to accurately model the complex handoff patterns in HWNs.

In this work, we establish a new analytical framework to quantify the rates of horizontal and vertical handoffs in $\mathrm{H}$ WNs with more general cell topologies and general UE movement patterns. In order to capture the spatial randomness, conventional stochastic geometric analysis on HWNs usually models each tier of BSs as a homogeneous PPP. However, the PPP cannot capture the non-uniform and dependent aggregation of BSs in, for example, popular regions of the network where more BSs tend to be installed, as the positions of the points in PPP are independent of each other. Instead, we accommodate the non-uniform and dependent aggregation by modeling some BS tiers as Poisson cluster processes (PCPs) [32]. Each BS cluster includes multiple nearby BSs, and multiple BS clusters are randomly distributed in space. Furthermore, to model flexible scaling of cell sizes in different tiers, we consider the biased user association scheme (also called flexible user association) [7,20,29], in which each tier of BSs is assigned an association bias value, and a UE is associated with a BS that provides the largest biased received power. In this case, the resultant cell splitting is a tessellation generated by both PPPs and PCPs (see Fig. 1(a) for an example). Through our proposed stochastic and analytic geometric analysis, we derive exact expressions for the rates of all handoff types experienced by an active UE with arbitrary movement trajectory.

Our characterization of the handoff rates also provides important guidelines for system design. One example is the open problem of optimal tier selection. Consider the example in Fig. 1(a), where an active UE's trajectory is indicated by the magenta arrow. By choosing to access both tier-1 and tier-2 cells, the UE experiences two vertical handoffs. In contrast, as shown in Fig. 1(b), by choosing to access tier-1 cells only, it experiences one horizontal handoff only. However, in the latter case, the UE gives up the opportunity to access a tier-2 BS, which potentially has higher bandwidth and requires lower power consumption. Thus, a UE may choose to access small-cell BSs to improve data rate and power consumption, but this may also lead to more frequent vertical handoffs, which potentially deteriorates the service quality. The proposed handoff analysis can be used to provide design

guidelines for optimal tier selection, where the handoff rate plays a key role.

The rest of this paper is organized as follows. In Section 2 , we discuss the relation between our work and prior works. In Section 3, we describe the system model. In Section 4, we present theoretical analysis on different types of handoff rates. In Section 5, we study the optimal tier selection problem as an application scenario of handoff rate analysis. In Section 6 , we validate our analysis with simulation. Finally, conclusions are given in Section 7.

\section{RELATED WORKS}

In this section, we survey the related prior works on mobility modeling and handoff analysis. We first summarize two categories of mobility modeling techniques, based on either abstract queueing formulations or actual geometric cell patterns. We then discuss relevant handoff and association decision algorithms in the literature.

\subsection{Mobility Modeling with Queueing Systems}

One classical category of mobility analysis techniques in cellular networks employ queueing formulation, without explicitly modeling the geometric patterns of cell shapes in the networks. In these works, cells are modeled as queues containing active users, and handoffs are modeled as unit transfers between queues. For example, Ghosh et al. [15] studied the single-cell scenario using an $M / G / \infty$ queue. Kirsal et al. [21] studied one WLAN cell overlaying one $3 \mathrm{G}$ cell, and a two-queue model was proposed accordingly. For more general multicell networks, queueing network models have been proposed and analyzed in $[3,5,10,13]$. In contrast, we explicitly model the cell geometry in this work.

\subsection{Mobility Modeling with Cell Geometry}

In mobility analysis techniques that consider cell geometry, non-random regular grids are commonly employed for mathematical convenience. Traditional one-tier cellular network is commonly modeled as a hexagonal grid [27]. For HWN analysis, Anpalagan and Katzela [2] studied a twotier network by modeling small-cells as hexagons, and each macrocell as a cluster of neighbouring small-cells. Shenoy and Hartpence [28] studied a two-tier network by modeling WLAN small-cells as squares, and macrocells as larger squares, each covering $5 \times 5$ WLAN cells. Hasib and Fapojuwo [17] studied a two-tier cellular network including one hexagonal macrocell and a predetermined number of circular microcells.

To further capture the spatial randomness of network topologies, Lin et al. [23] conducted a pioneering study on the user mobility in a one-tier cellular network with randomly distributed BSs, where the BSs were modeled as a homogeneous PPP, and cell splitting was modeled as a standard Poisson Voronoi. In $[6,9]$, we extended the above study to the multitier case, where each tier of BSs was modeled as a homogeneous PPP, and the resultant cell splitting was modeled as a weighted Poisson Voronoi. The studies in $[6,9,23]$ follow conventional stochastic geometric analysis of HWNs, where the PPP is commonly used to model the distribution of BSs to capture their spatial randomness (e.g., $[1,11,12])$. However, in reality, often a higher density of BSs are installed in more populated regions. The PPP assumption does not apply in such a scenario. In order to resolve this issue, the PCP has been adopted as an alternative to capture the non- 
uniform and dependent aggregation of BSs $[16,19,33]$. We thus use the PCP model in our work. Note that previous studies $[16,19,33]$ only focused on the average throughput and outage performance for randomly placed but stationary UEs. In contrast, in our work we consider moving UEs and their handoff between PCP BSs, which is essential to performance analysis in a mobile network but has not been addressed in the existing literature.

\subsection{Handoff and Association Decisions}

Orthogonal to the scope of our work, there is also a large body of previous works that study handoff timing algorithm$\mathrm{s}$, without considering the random geometric patterns of UEs and BSs. One type of handoff decision algorithms employ threshold comparison of one or several metrics (e.g., received signal strength, network loading, bandwidth, etc.) $[22,25,26]$. Another type uses dynamic programming [31] or artificial intelligence techniques [18] to improve the effectiveness of handoff procedures. In our work, we do not explicitly specify the handoff timing. Instead, we derive handoff rates and the corresponding optimal tier-selection decisions through stochastic geometric analysis.

The optimization of tier association in HWNs has been studied using stochastic geometric analysis [7,20,29]. These works assume that the UEs are randomly placed but stationary. They focus on average performance metrics such as the mean data throughput or outage probability. They ignore the movement of UEs and the effect of handoffs, which are the main focus of our work.

\section{SYSTEM MODEL}

In this section, we describe the multi-tier network under consideration, along with how the UEs are associated with BSs and are handed off between BSs as they move.

\subsection{Multi-tier Network}

We consider an HWN with randomly distributed $K$ tiers of BSs. It includes $K_{P}$ Poisson tiers (P-tiers) and $K_{C}$ Poisson cluster tiers (C-tiers), so that $K=K_{P}+K_{C}$. Let $\mathcal{K}_{P}=\left\{1, \ldots, K_{P}\right\}$ denote the set of P-tiers, and $\mathcal{K}_{C}=$ $\left\{K_{P}+1, \ldots, K_{P}+K_{C}\right\}$ denote the set of C-tiers. Let $\mathcal{K}=\left\{1, \ldots, K_{P}+K_{C}\right\}$.

Let $\Phi_{k}$ denote the point process representing the locations of tier- $k$ BSs. If $k \in \mathcal{K}_{P}, \Phi_{k}$ is a homogeneous PPP with intensity of $\lambda_{k}$ on the two dimensional space $\mathbb{R}^{2}$. If $k \in \mathcal{K}_{C}$, $\Phi_{k}$ is a PCP defined as follows: First, cluster centers are generated as a parent point process $\Theta_{k}$, which is a homogeneous PPP with intensity $\mu_{k}$ on the two dimensional space $\mathbb{R}^{2}$. Second, for each parent point (cluster center) $\mathbf{x}$ in $\Theta_{k}$, a cluster of BSs form a child point process $\Omega_{k}(\mathbf{x})$, which is a PPP with intensity $\nu_{k}$ in the region $\mathcal{B}\left(\mathbf{x}, R_{k}\right)$, where $\mathcal{B}(\mathbf{x}, R)$ is defined as the disk region centered at $\mathbf{x}$ with radius $R$ throughout this paper. The overall $\mathrm{PCP} \Phi_{k}$ is the union of all child point processes, i.e., $\Phi_{k}=\bigcup_{\mathbf{x} \in \Theta_{k}} \Omega_{k}(\mathbf{x})$. Note that $\Phi_{k}$ does not include parent points $\Theta_{k}$. Given the location of some $\mathrm{BS} \mathbf{x}_{\mathrm{BS}}$ in a tier- $k \mathrm{PCP}$, let $\mathcal{C}\left(\mathbf{x}_{\mathrm{BS}}\right)$ denote the cluster center of the BS. Thus $\Omega_{k}\left(\mathcal{C}\left(\mathbf{x}_{\mathrm{BS}}\right)\right)$ is the cluster it belongs to.

\subsection{Biased User Association}

Different tiers of BSs transmit at different power levels. Let $P_{k}$ be the transmission power of tier- $k$ BSs. These power levels are fixed and given. If $P_{t}(\mathbf{x})$ is the transmission power from a BS at location $\mathbf{x}$ and $P_{r}(\mathbf{y})$ is the received power at location $\mathbf{y}$, we have $P_{r}(\mathbf{y})=\frac{P_{t}(\mathbf{x})}{|\mathbf{x}-\mathbf{y}|^{\gamma}}$, where $|\mathbf{x}-\mathbf{y}|^{\gamma}$ is the propagation loss function with $\gamma>2$.

We consider a general biased user association rule as follows [20,29]. Given that a UE is located at $\mathbf{y}$, it associates itself with the BS that provides the maximum biased received power as follows:

$$
\mathcal{B S}(\mathbf{y})=\arg \max _{\mathbf{x} \in \Phi_{k}, \forall k} B_{k} P_{k}|\mathbf{x}-\mathbf{y}|^{-\gamma},
$$

where $\mathcal{B S}(\mathbf{y})$ denotes the location of the BS chosen to serve the UE, $P_{k}|\mathbf{x}-\mathbf{y}|^{-\gamma}$ is the received power from a tier- $k$ BS located at $\mathbf{x}$, and $B_{k}$ is the association bias, indicating the power preference of UEs toward tier- $k$ BSs.

As an example, the cell splitting resultant from biased user association is shown in Fig. 1(a) (a two-tier scenario including one P-tier and one C-tier). Let $\mathbf{T}^{(1)}$ denote the set of overall cell boundaries, and let $\mathbf{T}_{k j}^{(1)}$ denote the set of boundaries between tier- $k$ cells and tier- $j$ cells, which is also referred to as the set of type $k-j$ cell boundaries in this paper. Note that $\mathbf{T}_{k j}^{(1)}$ and $\mathbf{T}_{j k}^{(1)}$ are equivalent.

Note that for $B_{1}, B_{2}, \ldots, B_{K}$, their effects remain the same if we multiply all of them by the same positive constant. For presentation convenience, we define $\beta_{k j}=\left(\frac{P_{k} B_{k}}{P_{j} B_{j}}\right)^{1 / \gamma}$. Clearly, $\beta_{k j}=\frac{1}{\beta_{j k}}$, and $\beta_{k k}=1$.

\subsection{UE Trajectory and Handoff Rate}

We aim to study the rates of all handoff types of some active UE moving in the network. Let $\mathcal{T}_{0}$ denote the trajectory of the UE, which is of finite length. In this paper, a handoff made from a tier- $k$ cell to a tier- $j$ cell is called a type $k-j$ handoff. The total number of type $k-j$ handoffs along $\mathcal{T}_{0}$ is denoted by $\mathcal{N}_{k j}\left(\mathcal{T}_{0}, \mathbf{T}_{k j}^{(1)}\right)$.

Note that if $j \neq k$, a type $k$ - $j$ (vertical) handoff is not equivalent to a type $j-k$ handoff. When the UE crosses some type $k$ - $j$ boundary, either a type $k-j$ or a type $j-k$ handoff is made, depending on its direction of movement. Thus, the number of type $k$ - $j$ plus type $j$ - $k$ handoffs is equal to the number of intersections of $\mathcal{T}_{0}$ and $\mathbf{T}_{k j}^{(1)}$, which is denoted by $\mathcal{N}\left(\mathcal{T}_{0}, \mathbf{T}_{k j}^{(1)}\right)$. In other words, we have $\mathcal{N}\left(\mathcal{T}_{0}, \mathbf{T}_{k j}^{(1)}\right)=$ $\mathcal{N}_{k j}\left(\mathcal{T}_{0}, \mathbf{T}_{k j}^{(1)}\right)+\mathcal{N}_{j k}\left(\mathcal{T}_{0}, \mathbf{T}_{k j}^{(1)}\right) . \quad$ If $j=k, \mathcal{N}\left(\mathcal{T}_{0}, \mathbf{T}_{k k}^{(1)}\right)=$ $\mathcal{N}_{k k}\left(\mathcal{T}_{0}, \mathbf{T}_{k k}^{(1)}\right)$ indicates the number of type $k-k$ (horizontal) handoffs. We obtain the overall number of handoffs by adding the numbers of all types of handoffs.

\section{HANDOFF RATE ANALYSIS}

The proposed analysis of handoff rates consists of a progressive sequence of four components, which are described in the following subsections.

\subsection{Length Intensity of Cell Boundaries}

Handoffs occur at the intersections of the active UE's trajectory and cell boundaries. In order to track the number of intersections, we need to first study the length intensity of different types of cell boundaries $\mathbf{T}_{k j}^{(1)}$, which is defined as the expected length of $\mathbf{T}_{k j}^{(1)}$ in a unit square.

The set of cell boundaries $\mathbf{T}_{k j}^{(1)}$ is generated by all $K$ tiers of BSs $\Phi_{1}, \Phi_{2}, \ldots, \Phi_{K}$. It corresponds to the set of points on $\mathbb{R}^{2}$ where the same biased power level is received from 
a tier- $k$ BS and a tier- $j$ BS, and this biased received power level is greater than those from any other BS in all tiers. Mathematically, $\mathbf{T}_{k j}^{(1)}$ is expressed as

$$
\begin{aligned}
\mathbf{T}_{k j}^{(1)} & =\left\{\mathbf{x} \mid \exists \mathbf{x}_{1} \in \Phi_{k}, \mathbf{x}_{2} \in \Phi_{j}, \mathbf{x}_{1} \neq \mathbf{x}_{2} \text {, s.t. } P_{r}=\frac{P_{k} B_{k}}{\left|\mathbf{x}_{1}-\mathbf{x}\right|^{\gamma}}\right. \\
& \left.=\frac{P_{j} B_{j}}{\left|\mathbf{x}_{2}-\mathbf{x}\right|^{\gamma}} \text {, and } \forall i \in \mathcal{K}, \mathbf{y} \in \Phi_{i}, P_{r} \geq \frac{P_{i} B_{i}}{|\mathbf{y}-\mathbf{x}|^{\gamma}}\right\} .
\end{aligned}
$$

A main challenge in this work is in characterizing handoff rates across the highly irregular cell boundaries generated by BSs that form PCPs. If we consider the cell boundaries within tier- $k$, and tier- $k$ is a C-tier (i.e., $k \in \mathcal{K}_{C}$ ), then we can further classify the set of cell boundaries $\mathbf{T}_{k k}^{(1)}$ into the set of intra-cluster cell boundaries (i.e., the two BSs that provide the largest biased received power belong to the same cluster), which is denoted by $\mathbf{T}_{k k, i n}^{(1)}$, and the set of inter-cluster cell boundaries (i.e., the two BSs belong to two different clusters), which is denoted by $\mathbf{T}_{k k, \text { out }}^{(1)}$. We formally express

$$
\begin{aligned}
\mathbf{T}_{k k, i n}^{(1)}= & \left\{\mathbf{x} \mid \exists \mathbf{x}_{1} \in \Phi_{k}, \mathbf{x}_{2} \in \Phi_{k}, \mathbf{x}_{1} \neq \mathbf{x}_{2}, \mathcal{C}\left(\mathbf{x}_{1}\right)=\mathcal{C}\left(\mathbf{x}_{2}\right)\right. \\
& \text { s.t. } P_{r}=\frac{P_{k} B_{k}}{\left|\mathbf{x}_{1}-\mathbf{x}\right|^{\gamma}}=\frac{P_{j} B_{j}}{\left|\mathbf{x}_{2}-\mathbf{x}\right|^{\gamma}} \\
& \text { and } \left.\forall i \in \mathcal{K}, \mathbf{y} \in \Phi_{i}, P_{r} \geq \frac{P_{i} B_{i}}{|\mathbf{y}-\mathbf{x}|^{\gamma}}\right\}
\end{aligned}
$$

Similarly, by replacing $\mathcal{C}\left(\mathbf{x}_{1}\right)=\mathcal{C}\left(\mathbf{x}_{2}\right)$ by $\mathcal{C}\left(\mathbf{x}_{1}\right) \neq \mathcal{C}\left(\mathbf{x}_{2}\right)$ in (3), we have the expression of $\mathbf{T}_{k k, \text { out }}^{(1)}$.

Note that we have $\mathbf{T}_{k k}^{(1)}=\mathbf{T}_{k k, \text { in }}^{(1)} \cup \mathbf{T}_{k k, \text { out }}^{(1)}$. Also, if $k, j \in$ $\mathcal{K}_{C}$ and $k \neq j$, we cannot classify $\mathbf{T}_{k j}^{(1)}$ in the same way, as the cell boundary is surely formed by two BSs in two different clusters in two different tiers.

Let $\mu_{1}\left(\mathbf{T}_{k j}^{(1)}\right)$ denote the length intensity of $\mathbf{T}_{k j}^{(1)}$, which is the expected length of $\mathbf{T}_{k j}^{(1)}$ in a unit square. Because $\Phi_{1}, \ldots, \Phi_{K}$ are stationary, $\mathbf{T}_{k j}^{(1)}$ is also stationary, and thus the unit square could be arbitrarily picked on $\mathbb{R}^{2}$. Hence, we have

$$
\mu_{1}\left(\mathbf{T}_{k j}^{(1)}\right)=\mathbb{E}\left(\left|\mathbf{T}_{k j}^{(1)} \bigcap[0,1)^{2}\right|_{1}\right)
$$

where $|L|_{1}$ denotes the length of a collection of curves $L$ (i.e., one-dimensional Lebesgue measure of $L$ ). Similarly, if $k \in \mathcal{K}_{C}$,

$$
\mu_{1}\left(\mathbf{T}_{k k, x}^{(1)}\right)=\mathbb{E}\left(\left|\mathbf{T}_{k k, x}^{(1)} \bigcap[0,1)^{2}\right|_{1}\right)
$$

where the subscript " $x$ " indicates either "in" or "out". Note that $\mu_{1}\left(\mathbf{T}_{k k}^{(1)}\right)=\mu_{1}\left(\mathbf{T}_{k k, \text { in }}^{(1)}\right)+\mu_{1}\left(\mathbf{T}_{k k, \text { out }}^{(1)}\right)$.

\section{2 $\Delta d$-Extended Cell Boundaries}

It is difficult to directly quantify the one-dimensional measures $\mu_{1}\left(\mathbf{T}_{k j}^{(1)}\right), \mu_{1}\left(\mathbf{T}_{k k, \text { in }}^{(1)}\right)$, and $\mu_{1}\left(\mathbf{T}_{k k, \text { out }}^{(1)}\right)$ on the twodimensional plane. Instead, we first introduce the set of $\Delta d$ extended cell boundaries, which extends the one-dimensional measures to two-dimensional measures.
The $\Delta d$-extended cell boundaries of $\mathbf{T}_{k j}^{(1)}$, denoted by $\mathbf{T}_{k j}^{(2)}$ $(\Delta d)$, is defined as

$$
\mathbf{T}_{k j}^{(2)}(\Delta d)=\left\{\mathbf{x} \mid \exists \mathbf{y} \in \mathbf{T}_{k j}^{(1)}, \text { s.t. }|\mathbf{x}-\mathbf{y}|<\Delta d\right\} .
$$

In other words, $\mathbf{T}_{k j}^{(2)}(\Delta d)$ is the $\Delta d$-neighbourhood of $\mathbf{T}_{k j}^{(1)}$. A point is in $\mathbf{T}_{k j}^{(2)}(\Delta d)$ if and only if its (shortest) distance to $\mathbf{T}_{k j}^{(1)}$ is less than $\Delta d$. Similarly, $\mathbf{T}_{k k, i n}^{(2)}(\Delta d)$ and $\mathbf{T}_{k k, \text { out }}^{(2)}(\Delta d)$ are defined as the $\Delta d$-neighbourhoods of $\mathbf{T}_{k k, \text { in }}^{(1)}$ and $\mathbf{T}_{k k, \text { out }}^{(1)}$ respectively.

The area intensity of $\mathbf{T}_{k j}^{(2)}(\Delta d)$ is defined as the expected area of $\mathbf{T}_{k j}^{(2)}(\Delta d)$ in a unit square:

$$
\mu_{2}\left(\mathbf{T}_{k j}^{(2)}(\Delta d)\right)=\mathbb{E}\left(\left|\mathbf{T}_{k j}^{(2)}(\Delta d) \bigcap[0,1)^{2}\right|\right),
$$

where $|S|$ denotes the area of some region $S$ (i.e., two-dimensional Lebesgue measure of $S$ ).

Because $\Phi_{1}, \Phi_{2} \ldots, \Phi_{K}$ are stationary and isotropic, $\mathbf{T}_{k j}^{(2)}(\Delta d)$ is also stationary and isotropic. As a result, given a reference UE located at $\mathbf{0}$, the area intensity of $\mathbf{T}_{k j}^{(2)}(\Delta d)$ is equal to the probability that the reference $\mathrm{UE}$ at $\mathbf{0}$ is in $\mathbf{T}_{k j}^{(2)}(\Delta d)$.

$$
\mu_{2}\left(\mathbf{T}_{k j}^{(2)}(\Delta d)\right)=\mathbb{P}\left(\mathbf{0} \in \mathbf{T}_{k j}^{(2)}(\Delta d)\right) .
$$

Similarly, if $k \in \mathcal{K}_{C}$,

$$
\mu_{2}\left(\mathbf{T}_{k k, x}^{(2)}(\Delta d)\right)=\mathbb{P}\left(\mathbf{0} \in \mathbf{T}_{k k, x}^{(2)}(\Delta d)\right),
$$

where the subscript " $x$ " indicates either "in" or "out".

We observe that the probabilities in (8)-(9) are analytically tractable, which will be presented in the next subsection.

\subsection{Derivation of Area Intensities}

In this subsection, we present the derivation of $\mathbb{P}(\mathbf{0} \in$ $\left.\mathbf{T}_{k j}^{(2)}(\Delta d)\right)$. It consists of a progressive sequence of four steps. In the first step, we recall a few important properties of two intersecting circles, which will be frequently used in the subsequent steps. In the second step, we study the distribution of the distance from the reference $U E$ at $\mathbf{0}$ to the BS it is associated with, which is referred to as the reference $B S$ throughout the rest of this paper. In the third step, we study the conditional probability of $\mathbf{0} \in \mathbf{T}_{k j}^{(2)}(\Delta d)$ given the distance from the reference UE to the reference BS. Based on step two and step three, we derive the unconditioned probability of $\mathbf{0} \in \mathbf{T}_{k j}^{(2)}(\Delta d)$ in the fourth step.

\subsubsection{Geometric Patterns of Two Intersecting Circles}

We first recall a few important properties of two intersecting circles (as shown in Fig. 2), which will be frequently used in the subsequent steps to derive $\mathbb{P}\left(\mathbf{0} \in \mathbf{T}_{k j}^{(2)}(\Delta d)\right)$. Let $r_{1}$ and $r_{2}$ be the radii of these circles, and $r$ be the distance between their centers. We assume $\left|r_{1}-r_{2}\right| \leq r \leq r_{1}+r_{2}$.

As labeled in Fig. 2, let $C\left(r_{1}, r_{2}, r\right)$ denote the area of the overlapping part of the two circles, $L\left(r_{1}, r_{2}, r\right)$ denote the arc length of circle 1 covered by circle 2 , and $\theta_{m}\left(r_{1}, r_{2}, r\right)$ denote the half central angle corresponding to the arc. The expressions of $C(\cdot), L(\cdot)$, and $\theta_{m}(\cdot)$ are all in closed form, which are shown in Appendix 8.1.

\subsubsection{Distance Distribution of Reference UE-BS Pair}

Let $\mathcal{R}_{k}$ denote the distance from $\mathbf{0}$ to the nearest tier- $k$ BS. If $k \in \mathcal{K}_{P}$, i.e., $\Phi_{k}$ is a PPP, by the Markovian property 


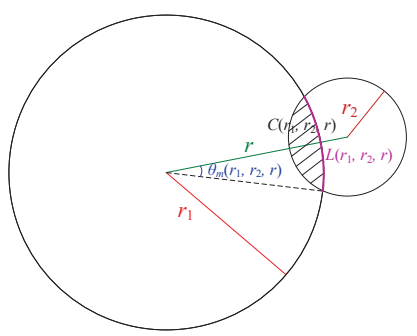

Figure 2: Geometric patterns of two intersecting circles.

of PPPs, it is straightforward to derive the complementary cumulative distribution function (ccdf) and probability density function (pdf) of $\mathcal{R}_{k}$ :

$$
\begin{aligned}
\operatorname{ccdf}_{\mathcal{R}_{k}}\left(R_{0}\right) & =\mathbb{P}\left(\mathcal{R}_{k}>R_{0}\right)=\exp \left(-\pi R_{0}^{2} \lambda_{k}\right), \\
\operatorname{pdf}_{\mathcal{R}_{k}}\left(R_{0}\right) & =2 \pi R_{0} \lambda_{k}\left(-\pi R_{0}^{2} \lambda_{k}\right) .
\end{aligned}
$$

If $k \in \mathcal{K}_{C}$, the ccdf of $\mathcal{R}_{k}$ is more complex due to the dependent and non-uniform aggregation of points in PCP. It can be shown that

$$
\begin{aligned}
& \operatorname{ccdf}_{\mathcal{R}_{k}}\left(R_{0}\right) \triangleq \mathbb{P}\left(\mathcal{R}_{k}>R_{0}\right)= \\
& \left\{\begin{array}{r}
\exp \left[-\pi\left(R_{0}-R_{k}\right)^{2} \mu_{k}\left(1-e^{-\pi R_{k}^{2} \nu_{k}}\right)\right. \\
\left.-\int_{R_{0}-R_{k}}^{R_{0}+R_{k}} 2 \pi r \mu_{k}\left(1-e^{-C\left(R_{0}, R_{k}, r\right) \nu_{k}}\right) \mathrm{d} r\right] \\
\text { if } R_{0} \geq R_{k}, \\
\exp \left[-\pi\left(R_{0}-R_{k}\right)^{2} \mu_{k}\left(1-e^{-\pi R_{0}^{2} \nu_{k}}\right)\right. \\
\left.-\int_{R_{k}-R_{0}}^{R_{k}+R_{0}} 2 \pi r \mu_{k}\left(1-e^{-C\left(R_{0}, R_{k}, r\right) \nu_{k}}\right) \mathrm{d} r\right] \\
\text { if } R_{0}<R_{k} .
\end{array}\right.
\end{aligned}
$$

By taking the first derivative, we find the pdf of $\mathcal{R}_{k}$ as

$$
\begin{gathered}
\operatorname{pdf}_{\mathcal{R}_{k}}\left(R_{0}\right)= \\
\left\{\begin{array}{c}
\exp \left[-\pi\left(R_{0}-R_{k}\right)^{2} \mu_{k}\left(1-e^{-\pi R_{k}^{2} \nu_{k}}\right)-\right. \\
\left.\int_{R_{0}-R_{k}}^{R_{0}+R_{k}} 2 \pi r \mu_{k}\left(1-e^{-C\left(R_{0}, R_{k}, r\right) \nu_{k}}\right) \mathrm{d} r\right] \\
\cdot \mu_{k} \int_{R_{0}-R_{k}}^{R_{0}+R_{k}} 2 \pi r e^{-\nu_{k} C\left(R_{0}, R_{k}, r\right)} \nu_{k} L\left(R_{0}, R_{k}, r\right) \mathrm{d} r \\
\text { if } R_{0} \geq R_{k}, \\
\exp \left[-\pi\left(R_{0}-R_{k}\right)^{2} \mu_{k}\left(1-e^{-\pi R_{0}^{2} \nu_{k}}\right)-\right. \\
\left.\int_{R_{k}-R_{0}}^{R_{k}+R_{0}} 2 \pi r \mu_{k}\left(1-e^{-C\left(R_{0}, R_{k}, r\right) \nu_{k}}\right) \mathrm{d} r\right] \\
\cdot \mu_{k}\left[2 \pi^{2}\left(R_{k}-R_{0}\right)^{2} R_{0} e^{-\pi R_{0}^{2} \nu_{k}} \nu_{k}\right. \\
\left.+\int_{R_{k}-R_{0}}^{R_{k}+R_{0}} 2 \pi r e^{-\nu_{k} C\left(R_{0}, R_{k}, r\right)} \nu_{k} L\left(R_{0}, R_{k}, r\right) \mathrm{d} r\right],
\end{array}\right.
\end{gathered}
$$

We note that the reference UE is placed at $\mathbf{0}$. Hence, $\mathcal{R}_{k}$ is also the distance from the reference UE to its nearest BS in tier- $k$. However, the reference UE is associated with the BS providing the largest biased received power (i.e., the reference $\mathrm{BS}$ ), which may not be the nearest BS. Let $\mathcal{R}$ denote the distance between the reference UE and the reference BS. The probability that the reference BS is a tier- $k$ BS and $\mathcal{R}$ is greater than some $R_{0}$ is derived as follows:

$$
\begin{aligned}
& \mathbb{P}\left[\mathcal{R}>R_{0}, \text { tier }=k\right]=\mathbb{P}\left[\mathcal{R}>R_{0} \mid \text { tier }=k\right] \mathbb{P}[\text { tier }=k] \\
= & \int_{R_{0}}^{\infty} \prod_{i=1, i \neq k}^{K} \mathbb{P}\left[\mathcal{R}_{i}>\beta_{i k} r\right] \operatorname{pdf}_{\mathcal{R}_{k}}(r) \mathrm{d} r .
\end{aligned}
$$

The last equality reflects the fact that the biased received power from the nearest BSs in all other tiers should not exceed that from the reference BS.
Let $\operatorname{pdf}_{\mathcal{R} \mid k}\left(R_{0}\right)$ denote the pdf of $\mathcal{R}$ given that the reference BS is in tier- $k$, and let $f_{\mathcal{R}, k}\left(R_{0}\right) \triangleq \operatorname{pdf}_{\mathcal{R} \mid k}\left(R_{0}\right) \mathbb{P}[$ tier $=$ $k]$, we have

$$
f_{\mathcal{R}, k}\left(R_{0}\right)=\prod_{i=1, i \neq k}^{K} \operatorname{ccdf}_{\mathcal{R}_{i}}\left(\beta_{i k} R_{0}\right) \cdot \operatorname{pdf}_{\mathcal{R}_{k}}\left(R_{0}\right) .
$$

\subsubsection{Conditional Probability of $\mathbf{0} \in \mathbf{T}_{k j}^{(2)}(\Delta d)$}

In this subsection, we study the conditional probabilities that the reference $\mathrm{UE}$ at $\mathbf{0}$ is in $\mathbf{T}_{k j}^{(2)}(\Delta d)$, given that it is associated with a tier- $k$ BS (reference BS) at a distance of $R_{0}$ from it, which is denoted as $\mathbb{P}\left(\mathbf{0} \in \mathbf{T}_{k j}^{(2)}(\Delta d) \mid \mathcal{R}=R_{0}\right.$, tier $\left.=k\right)$. By employing both analytic geometric and stochastic geometric tools, we derive the probability in different cases, given in Theorems 1-4 below. We note that the handoff rates for P-tiers are already known in the literature (e.g., $[6,9,23])$. These theorems additionally address boundaries between $\mathrm{P}$ tiers and C-tiers, boundaries between different C-tiers, and inter-cluster and intra-cluster boundaries within a C-tier.

For brevity, we define the following quantities that will be used extensively in the rest of this section:

$$
\mathcal{F}(\beta) \triangleq \frac{1}{\beta^{2}} \int_{0}^{\pi} \sqrt{\left(\beta^{2}+1\right)-2 \beta \cos (\theta)} \mathrm{d} \theta,
$$

and

$$
\mathcal{H}_{k j}\left(R_{0}\right) \triangleq \frac{1}{\pi} \mathcal{F}\left(\beta_{k j}\right) \beta_{k j} \frac{\operatorname{pdf}_{\mathcal{R}_{j}}\left(R_{0} \beta_{j k}\right)}{\operatorname{ccdf}_{\mathcal{R}_{j}}\left(R_{0} \beta_{j k}\right)} .
$$

TheOREM 1. If tier- $j$ is a P-tier, i.e., $j \in \mathcal{K}_{P}$, for all $k \in \mathcal{K}(k=j$ is allowed $)$, we have

$$
\begin{aligned}
& \mathbb{P}\left(\mathbf{0} \in \mathbf{T}_{k j}^{(2)}(\Delta d) \mid \mathcal{R}=R_{0}, \text { tier }=k\right) \\
= & 2 \lambda_{j} \Delta d R_{0} \mathcal{F}\left(\beta_{k j}\right)+\mathcal{O}\left(\Delta d^{2}\right) .
\end{aligned}
$$

Proof. See Appendix 8.2 for the proof.

Theorem 2. If tier- $j$ is a $C$-tier, i.e., $j \in \mathcal{K}_{C}$, for all $k \in \mathcal{K}, k \neq j$, we have

$$
\begin{aligned}
& \mathbb{P}\left(\mathbf{0} \in \mathbf{T}_{k j}^{(2)}(\Delta d) \mid \mathcal{R}=R_{0}, \text { tier }=k\right) \\
= & \mathcal{H}_{k j}\left(R_{0}\right) \Delta d+\mathcal{O}\left(\Delta d^{2}\right) .
\end{aligned}
$$

Proof. See Appendix 8.3 for the proof.

Theorem 3. If tier- $k$ is a $C$-tier, i.e., $k \in \mathcal{K}_{C}$, we have

$$
\begin{aligned}
& \mathbb{P}\left(\mathbf{0} \in \mathbf{T}_{k k, \text { out }}^{(2)}(\Delta d) \mid \mathcal{R}=R_{0}, \text { tier }=k\right) \\
= & \mathcal{H}_{k k}\left(R_{0}\right) \Delta d+\mathcal{O}\left(\Delta d^{2}\right) .
\end{aligned}
$$

Proof. Given the reference tier- $k$ BS located at $\mathbf{x}_{\mathrm{BS}}$, we know that $\mathbf{x}_{\mathrm{BS}}$ belongs to some BS cluster $\Omega_{k}\left(\mathcal{C}\left(\mathbf{x}_{\mathrm{BS}}\right)\right)$. Because the point process of cluster centers $\Theta_{k}$ is a PPP, by the Slivnyak Theorem [4], the set of all cluster centers other than $\mathcal{C}\left(\mathbf{x}_{\mathrm{BS}}\right)$ remain a PPP with the same statistics as $\Theta_{k}$. Consequently, if we denote the set of all tier- $k$ BSs other than the cluster $\Omega_{k}\left(\mathcal{C}\left(\mathbf{x}_{\mathrm{BS}}\right)\right)$ as $\Phi_{k}^{\prime}$, then $\Phi_{k}^{\prime}$ remains a $\mathrm{PCP}$ with the same statistics as $\Phi_{k}$. Because the set of intercluster cell boundaries $\mathbf{T}_{k k, \text { out }}^{(2)}(\Delta d)$ is generated by $\mathbf{x}_{\mathrm{BS}}$ and $\Phi_{k}^{\prime}$, and $\Phi_{k}^{\prime}$ is still a PCP, the proof of Theorem 3 is the same as that of Theorem 2 . 
The set of intra-cluster cell boundaries $\mathbf{T}_{k k, i n}^{(2)}(\Delta d)$ is generated by $\mathbf{x}_{\mathrm{BS}}$ and $\Omega_{k}\left(\mathcal{C}\left(\mathbf{x}_{\mathrm{BS}}\right)\right) \backslash\left\{\mathbf{x}_{\mathrm{BS}}\right\}$. We have the following theorem:

Theorem 4. If tier- $k$ is a C-tier, i.e., $k \in \mathcal{K}_{C}$, we have $\mathbb{P}\left(\mathbf{0} \in \mathbf{T}_{k k, i n}^{(2)}(\Delta d) \mid \mathcal{R}=R_{0}\right.$, tier $\left.=k\right)=G_{k}\left(R_{0}\right) \Delta d+\mathcal{O}\left(\Delta d^{2}\right)$, where $G_{k}\left(R_{0}\right)$ is expressed in (21) (shown on the next page).

The proof is omitted due to the space limitation.

\subsubsection{Unconditioned Probability of $\mathbf{0} \in \mathbf{T}_{k j}^{(2)}(\Delta d)$}

Through deconditioning on $\mathcal{R}$, we derive the unconditioned probabilities that the reference UE at $\mathbf{0}$ is in $\mathbf{T}_{k j}^{(2)}(\Delta d)$. If $k \neq j$, we have

$$
\begin{aligned}
& \mathbb{P}\left(\mathbf{0} \in \mathbf{T}_{k j}^{(2)}(\Delta d)\right) \\
= & \int_{0}^{\infty} \mathbb{P}\left(\mathbf{0} \in \mathbf{T}_{k j}^{(2)}(\Delta d) \mid \mathcal{R}=R_{0}, \text { tier }=k\right) f_{\mathcal{R}, k}\left(R_{0}\right) \mathrm{d} R_{0} \\
+ & \int_{0}^{\infty} \mathbb{P}\left(\mathbf{0} \in \mathbf{T}_{k j}^{(2)}(\Delta d) \mid \mathcal{R}=R_{0}, \text { tier }=j\right) f_{\mathcal{R}, j}\left(R_{0}\right) \mathrm{d} R_{0} .
\end{aligned}
$$

If $k \in \mathcal{K}_{P}$, we have

$$
\begin{aligned}
& \mathbb{P}\left(\mathbf{0} \in \mathbf{T}_{k k}^{(2)}(\Delta d)\right) \\
= & \int_{0}^{\infty} \mathbb{P}\left(\mathbf{0} \in \mathbf{T}_{k k}^{(2)}(\Delta d) \mid \mathcal{R}=R_{0}, \text { tier }=k\right) f_{\mathcal{R}, k}\left(R_{0}\right) \mathrm{d} R_{0} .
\end{aligned}
$$

If $k \in \mathcal{K}_{C}$, we have

$$
\begin{aligned}
& \mathbb{P}\left(\mathbf{0} \in \mathbf{T}_{k k, x}^{(2)}(\Delta d)\right) \\
= & \int_{0}^{\infty} \mathbb{P}\left(\mathbf{0} \in \mathbf{T}_{k k, x}^{(2)}(\Delta d) \mid \mathcal{R}=R_{0}, \text { tier }=k\right) f_{\mathcal{R}, k}\left(R_{0}\right) \mathrm{d} R_{0},
\end{aligned}
$$

where the subscript " $x$ " indicates either "in" or "out".

\subsection{From Area Intensities to Handoff Rates}

Next, we compute the handoff rates using the area intensities derived in Section 4.3. This involves two steps: (1) from area intensities $\mu_{2}\left(\mathbf{T}_{k j}^{(2)}(\Delta d)\right)$ to length intensities $\mu_{1}\left(\mathbf{T}_{k j}^{(1)}\right)$, and (2) from length intensities to handoff rates.

In the first step, we derive the length intensity $\mu_{1}\left(\mathbf{T}_{k j}^{(1)}\right)$ from the area intensity $\mu_{2}\left(\mathbf{T}_{k j}^{(2)}(\Delta d)\right)$. Following Section 3.2 in [14], we have

$$
\mu_{1}\left(\mathbf{T}_{y}^{(1)}\right)=\lim _{\Delta d \rightarrow 0} \frac{\mu_{2}\left(\mathbf{T}_{y}^{(2)}(\Delta d)\right)}{2 \Delta d},
$$

where the subscript " $y$ " indicates " $k j$ ", " $k k, i n$ ", or " $k k$, out".

Consequently, combining (15)-(25), we have

Case 1: If $k, j \in \mathcal{K}_{P}$ and $k \neq j$,

$$
\begin{aligned}
& \mu_{1}\left(\mathbf{T}_{k j}^{(1)}\right) \\
= & \int_{0}^{\infty} \prod_{i=1, i \neq k}^{K} \operatorname{ccdf}_{\mathcal{R}_{i}}\left(\beta_{i k} R_{0}\right) \operatorname{pdf}_{\mathcal{R}_{k}}\left(R_{0}\right) \lambda_{j} R_{0} \mathcal{F}\left(\beta_{k j}\right) \mathrm{d} R_{0} \\
+ & \int_{0}^{\infty} \prod_{i=1, i \neq j}^{K} \operatorname{ccdf}_{\mathcal{R}_{i}}\left(\beta_{i j} R_{0}\right) \operatorname{pdf}_{\mathcal{R}_{j}}\left(R_{0}\right) \lambda_{k} R_{0} \mathcal{F}\left(\beta_{j k}\right) \mathrm{d} R_{0} .
\end{aligned}
$$

Case 2: If $k \in \mathcal{K}_{P}$ we have

$$
\begin{aligned}
& \mu_{1}\left(\mathbf{T}_{k k}^{(1)}\right) \\
= & \int_{0}^{\infty} \prod_{i=1, i \neq k}^{K} \operatorname{ccdf}_{\mathcal{R}_{i}}\left(\beta_{i k} R_{0}\right) \operatorname{pdf}_{\mathcal{R}_{k}}\left(R_{0}\right) \lambda_{k} R_{0} \mathcal{F}(1) \mathrm{d} R_{0} .
\end{aligned}
$$

Case 3: If $k \in \mathcal{K}_{P}$ and $j \in \mathcal{K}_{C}, \mu_{1}\left(\mathbf{T}_{k j}^{(1)}\right)$ can be obtained by replacing the term $\lambda_{j} R_{0} \mathcal{F}\left(\beta_{k j}\right)$ with $\frac{1}{2} \mathcal{H}_{k j}\left(R_{0}\right)$ in (26).

Case 4: If $k, j \in \mathcal{K}_{C}$ and $k \neq j, \mu_{1}\left(\mathbf{T}_{k j}^{(1)}\right)$ can be obtained by replacing the term $\lambda_{j} R_{0} \mathcal{F}\left(\beta_{k j}\right)$ with $\frac{1}{2} \mathcal{H}_{k j}\left(R_{0}\right)$ and the term $\lambda_{k} R_{0} \mathcal{F}\left(\beta_{j k}\right)$ with $\frac{1}{2} \mathcal{H}_{j k}\left(R_{0}\right)$ in $(26)$.

Case 5: If $k \in \mathcal{K}_{C}$, by replacing the term $\lambda_{k} R_{0} \mathcal{F}(1)$ with $\frac{1}{2} \mathcal{H}_{k k}\left(R_{0}\right)$ and $\frac{1}{2} G_{k}\left(R_{0}\right)$ in $(27)$, we find the expressions of $\mu_{1}\left(\mathbf{T}_{k k, \text { out }}^{(1)}\right)$ and $\mu_{1}\left(\mathbf{T}_{k k, \text { in }}^{(1)}\right)$ respectively.

In the second step, we derive the expected number of handoffs of an active UE as follows:

THEOREM 5. Let $\mathcal{T}_{0}$ denote an arbitrary UE's trajectory on $\mathbb{R}^{2}$ with length $\left|\mathcal{T}_{0}\right|_{1}$. Then, the expected number of intersections between $\mathcal{T}_{0}$ and $\mathbf{T}_{k j}^{(1)}$ is

$$
\mathbb{E}\left(\mathcal{N}\left(\mathcal{T}_{0}, \mathbf{T}_{k j}^{(1)}\right)\right)=\frac{2}{\pi} \mu_{1}\left(\mathbf{T}_{k j}^{(1)}\right)\left|\mathcal{T}_{0}\right|_{1},
$$

and the expected number of type $k-j$ handoffs is

$$
\mathbb{E}\left(\mathcal{N}_{k j}\left(\mathcal{T}_{0}, \mathbf{T}_{k j}^{(1)}\right)\right)= \begin{cases}\frac{1}{2} \mathbb{E}\left(\mathcal{N}\left(\mathcal{T}_{0}, \mathbf{T}_{k j}^{(1)}\right)\right), & \text { if } k \neq j, \\ \mathbb{E}\left(\mathcal{N}\left(\mathcal{T}_{0}, \mathbf{T}_{k j}^{(1)}\right)\right), & \text { if } k=j\end{cases}
$$

Proof. We note that $\mathbf{T}_{k j}^{(1)}$ is a stationary and isotropic fibre process with length intensity $\mu_{1}\left(\mathbf{T}_{k j}^{(1)}\right)$. The proof follows the conclusions in Section 9.3 of [32].

Note that the expected number of type $k$ - $j$ handoffs is the same as the expected number of type $j-k$ handoffs, both of which are equal to half of $\mathbb{E}\left(\mathcal{N}\left(\mathcal{T}_{0}, \mathbf{T}_{k j}^{(1)}\right)\right)$.

Furthermore, let $v$ denote the instantaneous velocity of an active UE, and $H_{k j}(v)$ denote its type $k$ - $j$ handoff rate. We have the following Corollary from Theorem 5:

Corollary 1.

$$
H_{k j}(v)= \begin{cases}\frac{1}{\pi} \mu_{1}\left(\mathbf{T}_{k j}^{(1)}\right) v, & \text { if } k \neq j, \\ \frac{2}{\pi} \mu_{1}\left(\mathbf{T}_{k j}^{(1)}\right) v, & \text { if } k=j .\end{cases}
$$

Note that the above handoff rates are instantaneous rates. Hence, our analysis allows time-varying velocity for the UEs, in which case the handoff rates are also time varying.

\section{APPLICATION SCENARIO: OPTIMAL TIER SELECTION}

In this section, we study the problem of optimal tier selection, as an application scenario for the handoff rate analysis above. Note that previous works focusing on biased user association in HWNs usually assumed stationary UEs [7-9,20,24,29]. In contrast, in this section, a UE's velocity is accommodated as one important factor for its tier-selection decision. The optimal tier selections are individualized for different UEs and can be adaptive to a UE's changing velocity over time.

Suppose that an active UE chooses to connect only to a set of tiers $\mathcal{S}$ that is a subset of $\mathcal{K}$ and ignore other tiers. We assume that $1 \in \mathcal{S}$ (i.e., the UE always includes tier1 in its candidate set, which may correspond to macrocells in practice). Our goal is to derive the optimal selection of candidate tiers $\mathcal{S}$ among all subsets of $\mathcal{K}$.

Following (14), the probability that the active UE is associated with a tier- $k \mathrm{BS}$, for $k \in \mathcal{S}$, is

$$
A_{k, \mathcal{S}}=\mathbb{P}[\mathcal{R}>0, \text { tier }=k]
$$




$$
G_{k}\left(R_{0}\right)= \begin{cases}\frac{\int_{R_{0}-R_{k}}^{R_{0}+R_{k}} r \nu_{k} R_{0} \cdot\left(16 \theta_{m}\left(R_{0}, R_{k}, r\right)-16 \sin \theta_{m}\left(R_{0}, R_{k}, r\right)\right) \exp \left(-\nu_{k} C\left(R_{0}, R_{k}, r\right)\right) \mathrm{d} r}{\int_{R_{0}-R_{k}}^{R_{0}+R_{k}} 2 \theta_{m}\left(R_{0}, R_{k}, r\right) r \exp \left(-C\left(R_{0}, R_{k}, r\right) \nu_{k}\right) \mathrm{d} r}, & \text { if } R_{0} \geq R_{k}, \\ \frac{\pi\left(R_{k}-R_{0}\right)^{2} \exp \left(-\pi R_{0}^{2} \nu_{k}\right) \cdot 8 \nu_{k} R_{0}+\int_{R_{k}}^{R_{k}+R_{0}} r \nu_{k} R_{0} \cdot\left(16 \theta_{m}\left(R_{0}, R_{k}, r\right)-16 \sin \theta_{m}\left(R_{0}, R_{k}, r\right)\right) \exp \left(-\nu_{k} C\left(R_{0}, R_{k}, r\right)\right) \mathrm{d} r}{\pi\left(R_{k}-R_{0}\right)^{2} \exp \left(-\pi R_{0}^{2} \nu_{k}\right)+\int_{R_{k}-R_{0}}^{R_{k}+R_{0}} 2 \theta_{m}\left(R_{0}, R_{k}, r\right) r \exp \left(-C\left(R_{0}, R_{k}, r\right) \nu_{k}\right) \mathrm{d} r}, & \text { if } R_{0}<R_{k} .\end{cases}
$$

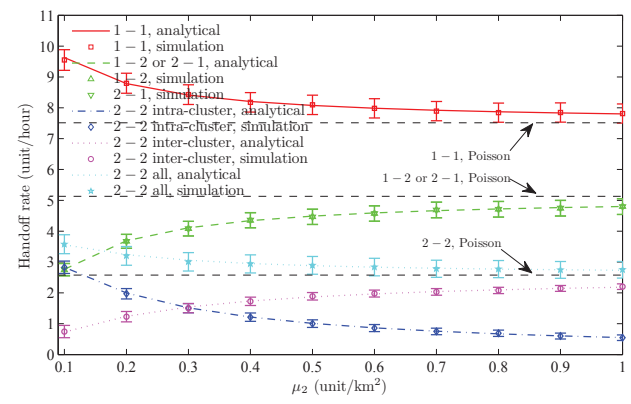

Figure 3: Accuracy of PCP handoff rate analysis under different $\mu_{2}$ values, for $\mu_{2} \cdot \nu_{2}=0.5$. For comparison, black dashed lines indicate analytical results assuming all PPP BSs.

$$
=\int_{0}^{\infty} \prod_{i \in \mathcal{S}, i \neq k} \operatorname{ccdf}_{\mathcal{R}_{i}}\left(\beta_{i k} R_{0}\right) \cdot \operatorname{pdf}_{\mathcal{R}_{k}}\left(R_{0}\right) \mathrm{d} R_{0} .
$$

We assume that the active UE is given some tier access benefit $\mathcal{U}_{k}$ per second if it is associated with a tier- $k$ BS. For example, such benefit may represent data rate, power consumption, and service charge at the tier. If the active UE's tier selection is $\mathcal{S}$, its overall tier access utility (per second) is

$$
U(\mathcal{S})=\sum_{k \in \mathcal{S}} \mathcal{U}_{k} A_{k, \mathcal{S}}
$$

Let $\mathcal{E}_{k j}$ be the expense for each type $k-j$ handoff. Such handoff expenses could be assigned arbitrarily. For example, the expense on a horizontal handoff $\mathcal{E}_{k k}$ is expected to be smaller than that of a vertical handoff $\mathcal{E}_{k j}(k \neq j)$. The average handoff expense (per second) given tier selection and velocity is computed as

$$
E(v, \mathcal{S})=\sum_{k, j \in \mathcal{S}} \mathcal{E}_{k j} H_{k j}(v, \mathcal{S})
$$

where $H_{k j}(v, \mathcal{S})$ are the handoff rates computed from (30) for the set of tiers $\mathcal{S}$.

Finally, the optimal tier selection under velocity $v$ is

$$
\mathcal{S}_{\text {opt }}(v)=\arg \max _{\mathcal{S} \in \mathbb{S}} U(\mathcal{S})-E(v, \mathcal{S}),
$$

where $\mathbb{S}$ is the set of all possible tier selections. Because the number of tiers $K$ is usually not high in reality (i.e., $K \leq 5$ ), the cardinality of $\mathbb{S}, 2^{K-1}$, is not large. Therefore, $\mathcal{S}_{\text {opt }}$ can be derived through comparing all possible tier selections.

\section{NUMERICAL STUDY}

In this section, we present simulation studies in Matlab to validate the accuracy and usefulness of the proposed analytical framework. In each round of simulation, multiple tiers of BSs are generated on a $20 \mathrm{~km} \times 20 \mathrm{~km}$ square. Then, we randomly generate 5 waypoints $\mathbf{X}_{1}, \mathbf{X}_{2}, \ldots, \mathbf{X}_{5}$ in

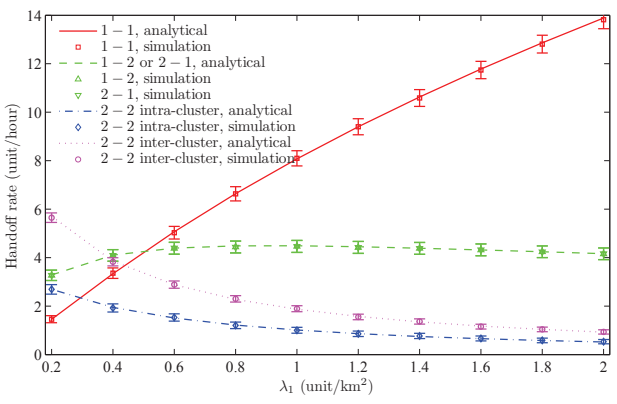

Figure 4: Handoff rates under different $\lambda_{1}$ values.

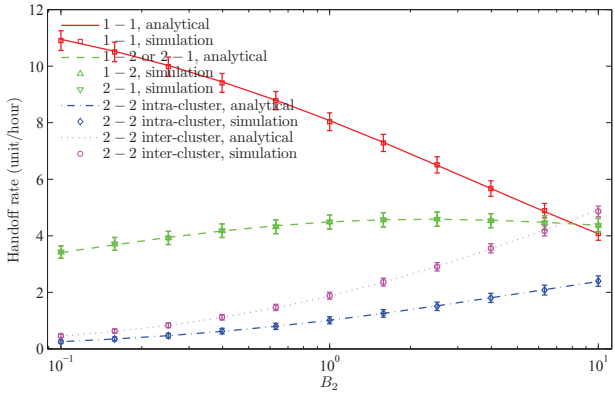

Figure 5: Handoff rates under different $B_{2}$ values.

the central $10 \mathrm{~km} \times 10 \mathrm{~km}$ square. The four line segments $\mathbf{X}_{1} \mathbf{X}_{2}, \mathbf{X}_{2} \mathbf{X}_{3}, \ldots, \mathbf{X}_{4} \mathbf{X}_{5}$ form the trajectory of an active UE in one round of simulation. By tracking which BSs the UE is associated with along its trajectory, we can obtain its handoff rates in this round of simulation. Each simulation data point is averaged over 2000 simulation rounds. Error bars in the figures show the $95 \%$ confidence intervals for simulation results.

Comparison with Simple PPP Modeling. First, we focus on a two-tier HWN with one P-tier (representing macrocell BSs) and one C-tier (representing clustered femtocell BSs). Tier-1 is the P-tier, with $\lambda_{1}=1 \mathrm{unit} / \mathrm{km}^{2}, P_{1}=30$ $\mathrm{dBm}$, and $B_{1}=1$; tier-2 is the C-tier, with $R_{2}=1 \mathrm{~km}$, $P_{2}=20 \mathrm{dBm}$, and $B_{2}=1 ; \gamma=3.5$; and $v=10 \mathrm{~km} /$ hour. In Fig. 3, we study the handoff rates under different $\mu_{2}$ values while maintaining a constant $\mu_{2} \nu_{2}=0.5$ (i.e., the overall tier-2 BS intensity is a constant $0.5 \pi$ unit $\left./ \mathrm{km}^{2}\right)$. For reference, we also show the handoff rates (black dashed lines) if tier-2 is replaced by a P-tier with the same BS intensity of $0.5 \pi$ unit $/ \mathrm{km}^{2}$. Fig. 3 shows that the actual handoff rates obtained from simulation match well with the proposed analysis, while they are far from the dashed lines when $\mu_{2}$ is small (and $\nu_{2}$ is large). This interesting observation suggest$\mathrm{s}$ that using a simple PPP to model the BSs as in $[6,9,23]$ can lead to substantial numerical errors in computing the handoff rates in HWNs with clustered BSs. The PPP model gives a close approximation only when the cluster intensities 


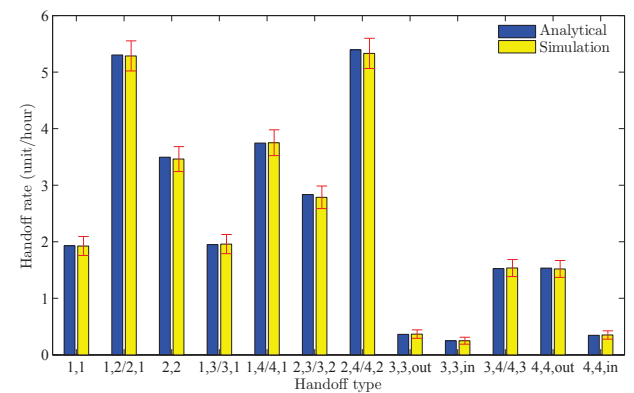

Figure 6: Handoff rates, two P-tiers and two C-tiers.

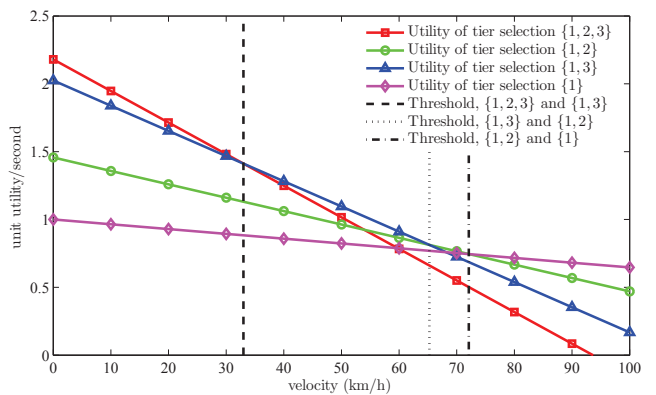

Figure 7: Optimal tier selection.

are high but per cluster BS intensities are low in the C-tier. In general, we observe that the actual handoff rates vary drastically as $\mu_{2}$ and $\nu_{2}$ change, which can be accounted for only by the proposed PCP analysis.

Effect of BS Densities and Association Bias Values. We further study the influence of different network parameters on the handoff rates. In Figs. 4 and 5, we consider a two-tier network with the same default parameter values as in Fig. 3, except $\mu_{2}=0.5$ unit $/ \mathrm{km}^{2}$ and $\nu_{2}=1 \mathrm{unit} / \mathrm{km}^{2}$.

Fig. 4 shows handoff rates under different tier-1 BS intensities $\lambda_{1}$. The figure illustrates that increasing $\lambda_{1}$ leads to a higher type 1-1 handoff rate but a lower type 2-2 handoff rate. Fig. 5 shows the handoff rates under different tier-2 association bias values $B_{2}$. We observe that increasing the association bias value of one tier has a similar effect as increasing the BS intensity of this tier, leading to a higher horizontal handoff rate within this tier, but lower handoff rates outside the tier. Moreover, both figures illustrate that the simulation results agree with the analytical results, demonstrating the correctness of our analysis for different types of handoff rates.

A More Complex Example. In Fig. 6, we study different types of handoff rates in a network with four tiers. The network parameters are as follows: Tier- 1 and tier- 2 are Ptiers, with $\left(\lambda_{1}, \lambda_{2}\right)=(1,1)$ unit $/ \mathrm{km}^{2}$; tier- 3 and tier- 4 are Ctiers, with $\left(\mu_{3}, \mu_{4}\right)=(0.5,1)$ unit $/ \mathrm{km}^{2},\left(R_{3}, R_{4}\right)=(0.9,1.1)$ unit $/ \mathrm{km}^{2}$, and $\left(\nu_{3}, \nu_{4}\right)=(1,0.5)$ unit $/ \mathrm{km}^{2} ;\left(P_{1}, P_{2}, P_{3}, P_{4}\right)=$ $(30,33,20,23) \mathrm{dBm} ;\left(B_{1}, B_{2}, B_{3}, B_{4}\right)=(1,1,1,1) ; \gamma=3.5$; and $v=10 \mathrm{~km} /$ hour. In order to avoid redundancy, we only show the sum rate of type $k-j$ and type $j-k(k \neq j)$ handoffs for easier inspection; the individual handoff rates are half of the sum handoff rate. We again observe that the simulation results agree with the analytical results, validating the correctness of our analysis. We further observe that the handoff rates of types $2-2,2-3 / 3-2$, and 2-4/4-2 are higher than those of $1-1,1-3 / 3-1$, and $1-4 / 4-1$ respectively, due to the higher transmission power of tier-2 BSs compared with tier-1 BSs. The handoff rates of types $4-4,1-4 / 4-1$, and 2-4/4-2 are higher than those of 3-3, 1-3/3-1, and 2-3/3-2 respectively, due to the higher transmission power and higher intensity of tier- 4 BSs, i.e., $\pi R_{4}^{2} \mu_{4} \nu_{4}$, compared with tier-3 BSs.

Tier Selection Optimization. Finally, we present an example of the optimal tier selection problem presented in Section 5. The network parameters are as follows: tier- 1 is a Ptier, with $\lambda_{1}=1$ unit $/ \mathrm{km}^{2}$; tier- 2 and tier-3 are C-tiers, with $\left(\mu_{2}, \mu_{3}\right)=(0.5,1)$ unit $/ \mathrm{km}^{2},\left(R_{2}, R_{3}\right)=(0.9,1.1)$ unit $/ \mathrm{km}^{2}$, and $\left(\nu_{2}, \nu_{3}\right)=(1,0.5)$ unit $/ \mathrm{km}^{2} ;\left(P_{1}, P_{2}, P_{3}\right)=(30,20,23)$ $\mathrm{dBm} ;\left(B_{1}, B_{2}, B_{3}\right)=(1,1,1)$; and $\gamma=3.5$. Tier access benefits are $\left(\mathcal{U}_{1}, \mathcal{U}_{2}, \mathcal{U}_{3}\right)=(1,3,3.5)$ unit utility/second. The horizontal handoff expenses are $\left(\mathcal{E}_{11}, \mathcal{E}_{22}, \mathcal{E}_{33}\right)=(10,15,15)$ unit utility per handoff, and the vertical handoff expenses are $\left(\mathcal{E}_{12}, \mathcal{E}_{21}, \mathcal{E}_{13}, \mathcal{E}_{31}, \mathcal{E}_{23}, \mathcal{E}_{32}\right)=(30,30,50,50,50,50)$ unit utility per handoff.

Given a specific tier selection, the overall tier access benefit does not change with the UE's velocity, but the handoff expense increases linearly. Therefore, the overall utility linearly decreases with the UE's velocity. As shown in Fig. 7, when the UE's velocity is low, tier selection $\{1,2,3\}$ is preferred since the handoff expense is relatively low compared with its tier access benefit. When the UE's velocity is high, tier selection $\{1\}$ is preferred, because handoff expense dominates tier access benefit, and tier selection $\{1\}$ has the smallest handoff expense. Overall, the UE's velocity can be divided into four regions: tier selection $\{1,2,3\}$ is optimal when the velocity is in $[0,33.00) \mathrm{km} /$ hour; tier selection $\{1,3\}$ is optimal when the velocity is in $[33.00,65.27$ ) $\mathrm{km} /$ hour; tier selection $\{1,2\}$ is optimal when the velocity is in $[65.27,72.10) \mathrm{km} /$ hour; tier selection $\{1\}$ is optimal when the velocity is in $[72.10, \infty) \mathrm{km} /$ hour.

\section{CONCLUSIONS}

In this work, we present a stochastic geometric framework to study user mobility in multi-tier HWNs. Each tier of BSs is modeled as either a PPP or a PCP. We are able to capture (1) the spatial randomness of BSs, (2) the non-uniform and dependent aggregation of BSs, and (3) the various scales of cell sizes. Theoretical expressions for all types of handoff rates experienced by an active UE with arbitrary movement trajectory are derived. Based on these results, optimal tier selection considering both the handoff expense and tier access benefit is studied. Finally, extensive simulations are conducted, validating the accuracy and usefulness of our analytical results.

\section{APPENDIX}

\subsection{Expressions Related to Two Intersecting Circles}

By applying basic analytical geometric tools, it can be shown that

$$
\begin{gathered}
C\left(r_{1}, r_{2}, r\right)=\arccos \left(\left(r_{1}^{2}+r^{2}-r_{2}^{2}\right) /\left(2 r_{1} r\right)\right) \cdot r_{1}^{2} \\
+\arccos \left(\left(r_{2}^{2}+r^{2}-r_{1}^{2}\right) /\left(2 r_{2} r\right)\right) \cdot r_{2}^{2} \\
-2 \sqrt{\left(\left(r_{1}+r_{2}+r\right) / 2\right)\left(\left(r_{1}+r_{2}+r\right) / 2-r_{1}\right)}
\end{gathered}
$$




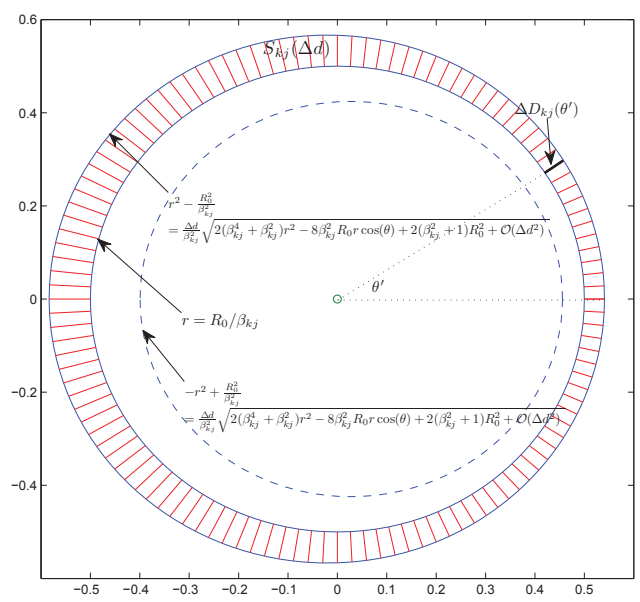

Figure 8: The region (shaded part) of $S_{k j}(\Delta d)$.

$$
\begin{gathered}
\cdot \sqrt{\left(\left(r_{1}+r_{2}+r\right) / 2-r_{2}\right)\left(\left(r_{1}+r_{2}+r\right) / 2-r\right)}, \\
L\left(r_{1}, r_{2}, r\right)=2 r_{1} \arccos \left(\left(r_{1}^{2}+r^{2}-r_{2}^{2}\right) /\left(2 r_{1} r\right)\right), \\
\theta_{m}\left(r_{1}, r_{2}, r\right)=\arccos \left(\left(r_{1}^{2}+r^{2}-r_{2}^{2}\right) /\left(2 r_{1} r\right)\right) .
\end{gathered}
$$

\subsection{Proof of Theorem 1}

Proof. We study the probability $\mathbb{P}\left(\mathbf{0} \in \mathbf{T}_{k j}^{(2)}(\Delta d) \mid \mathcal{R}=\right.$ $R_{0}$, tier $\left.=k\right)$. Without loss of generality, we assume the reference BS is located at $\mathbf{R}_{0}=\left(R_{0}, 0\right)$. Note that because the reference UE receives the highest biased power level from the reference BS, for all other tiers, there are no tier- $j$ BSs located within $\mathcal{B}\left(\mathbf{0}, \frac{R_{0}}{\beta_{k_{j}}}\right)$.

Let $\mathbf{x}_{0}=\left(x_{0}, y_{0}\right)$ denote the position of some tier- $j$ BS (other than the reference BS if $j=k$ ). Let $\mathcal{T}\left(\mathbf{R}_{0}, \mathbf{x}_{0}, \beta_{k j}\right.$ ) denote the curve satisfying the following condition:

$$
\begin{array}{r}
\mathcal{T}\left(\mathbf{R}_{0}, \mathbf{x}_{0}, \beta_{k j}\right)=\left\{(x, y) \mid \frac{P_{k} B_{k}}{\left(\left(x-R_{0}\right)^{2}+y^{2}\right)^{\gamma / 2}}=\right. \\
\left.\frac{P_{j} B_{j}}{\left(\left(x-x_{0}\right)^{2}+\left(y-y_{0}\right)^{2}\right)^{\gamma / 2}}\right\} .
\end{array}
$$

Note that $\mathbf{0} \in \mathbf{T}_{k j}^{(2)}(\Delta d)$ is equivalent to the event that the distance from $\mathbf{0}$ to $\mathcal{T}\left(\mathbf{R}_{0}, \mathbf{x}_{0}, \beta_{k j}\right)$ is less than $\Delta d$.

In the following, we discuss three cases separately: $\beta_{k j}>$ $1, \beta_{k j}=1$, and $\beta_{k j}<1$.

Case 1: $\beta_{k j}>1$. In this case, $\mathcal{T}\left(\mathbf{R}_{0}, \mathbf{x}_{0}, \beta_{k j}\right)$ is a circle centered at $\left(\frac{\beta_{k j}^{2} x_{0}-R_{0}}{\beta_{k j}^{2}-1}, \frac{\beta_{k j}^{2} y_{0}}{\beta_{k j}^{2}-1}\right)$ with radius $\frac{\beta_{k j} \sqrt{\left(R_{0}^{2}+x_{0}^{2}+y_{0}^{2}-2 x_{0} R_{0}\right)}}{\left(\beta_{k j}^{2}-1\right)}$. Thus, the distance from $\mathbf{0}$ to $\mathcal{T}\left(\mathbf{R}_{0}\right.$, $\left.\mathbf{x}_{0}, \beta_{k j}\right)$ is

$$
\begin{aligned}
& d\left(\mathbf{R}_{0}, \mathbf{x}_{0}, \beta_{k j}\right) \\
= & \left|\frac{\sqrt{\left(\beta_{k j}^{2} x_{0}-R_{0}\right)^{2}+\left(\beta_{k j}^{2} y_{0}\right)^{2}}-\sqrt{\beta_{k j}^{2}\left(R_{0}^{2}+x_{0}^{2}+y_{0}^{2}-2 x_{0} R_{0}\right)}}{\left(\beta_{k j}^{2}-1\right)}\right| .
\end{aligned}
$$

As a consequence, $\mathbf{0} \in \mathbf{T}_{k j}^{(2)}(\Delta d)$ iff $d\left(\mathbf{R}_{0}, \mathbf{x}_{0}, \beta_{k j}\right)<\Delta d$, or equivalently, $\mathbf{x}_{0} \in \widetilde{S}_{k j}(\Delta d)$, where

$$
\widetilde{S}_{k j}(\Delta d)=\left\{\mathbf{x}_{0} \mid d\left(\mathbf{R}_{0}, \mathbf{x}_{0}, \beta_{k j}\right)<\Delta d\right\} .
$$

After mathematical manipulations on (40), and converting $\left(x_{0}, y_{0}\right)$ into polar coordinates $(r, \theta)$, we have

$\widetilde{S}_{k j}(\Delta d)=\left\{(r, \theta)|| r^{2}-\frac{R_{0}^{2}}{\beta_{k j}^{2}} \mid<\frac{\Delta d}{\beta_{k j}^{2}}\right.$.

$\left.\sqrt{2\left(\beta_{k j}^{4}+\beta_{k j}^{2}\right) r^{2}-8 \beta_{k j}^{2} R_{0} r \cos (\theta)+2\left(\beta_{k j}^{2}+1\right) R_{0}^{2}+\mathcal{O}\left(\Delta d^{2}\right)}\right\}$.

Note that there are no tier- $j$ BSs located inside $\mathcal{B}\left(\mathbf{0}, \frac{R_{0}}{\beta_{k j}}\right)$. Let $S_{k j}(\Delta d)=\widetilde{S}_{k j}(\Delta d) \cap \overline{\mathcal{B}\left(\mathbf{0}, R_{0} / \beta_{k j}\right)}$, where $\overline{\mathcal{B}(\cdot)} \triangleq \mathbb{R}^{2} \backslash \mathcal{B}(\cdot)$ is the complement of $\mathcal{B}(\cdot)$. As a result, $\mathbf{0} \in \mathbf{T}_{k j}^{(2)}(\Delta d)$ iff $\mathbf{x}_{0} \in S_{k j}(\Delta d)$, where

$S_{k j}(\Delta d)=\left\{(r, \theta) \mid r \geq \frac{R_{0}}{\beta_{k j}}\right.$ and $\left|r^{2}-\frac{R_{0}^{2}}{\beta_{k j}^{2}}\right|<\frac{\Delta d}{\beta_{k j}^{2}}$.

$\left.\sqrt{2\left(\beta_{k j}^{4}+\beta_{k j}^{2}\right) r^{2}-8 \beta_{k j}^{2} R_{0} r \cos (\theta)+2\left(\beta_{k j}^{2}+1\right) R_{0}^{2}+\mathcal{O}\left(\Delta d^{2}\right)}\right\}$.

According to (42), $S_{k j}(\Delta d)$ corresponds to a non-concentric "ring" region (shaded area) shown in Fig. 8. We can observe that $\forall(r, \theta) \in S_{k j}(\Delta d), r=\frac{R_{0}}{\beta_{k j}}+\mathcal{O}(\Delta d)$. Substituting it into (42) gives

$$
\begin{aligned}
& S_{k j}(\Delta d)=\left\{(r, \theta) \mid r \geq \frac{R_{0}}{\beta_{k j}} \text { and }\left|r^{2}-\frac{R_{0}^{2}}{\beta_{k j}^{2}}\right|<\right. \\
& \left.\frac{2 \Delta d R_{0}}{\beta_{k j}^{2}} \sqrt{\left(\beta_{k j}^{2}+1\right)-2 \beta_{k j} \cos (\theta)}+\mathcal{O}\left(\Delta d^{2}\right)\right\} .
\end{aligned}
$$

The area of $S_{k j}(\Delta d)$ is

$$
\left|S_{k j}(\Delta d)\right|=\frac{2 \Delta d R_{0}}{\beta_{k j}^{2}} \int_{0}^{\pi} \sqrt{\left(\beta_{k j}^{2}+1\right)-2 \beta_{k j} \cos (\theta)} \mathrm{d} \theta+\mathcal{O}\left(\Delta d^{2}\right) .
$$

Given the reference UE and BS, it can be shown that $\Phi_{j}{ }^{1}$ is a PPP with intensity 0 in $\mathcal{B}\left(\mathbf{0}, \frac{R_{0}}{\beta_{k j}}\right)$ and intensity $\lambda_{j}$ in $\overline{\mathcal{B}\left(\mathbf{0}, R_{0} / \beta_{k j}\right)}[4]$. Because $\mathbb{P}\left(\mathbf{0} \in \mathbf{T}_{k j}^{(2)}(\Delta d) \mid \mathcal{R}=R_{0}\right.$, tier $=$ $k)$ is equal to the probability that there is at least one point of $\Phi_{j}$ in $S_{k j}(\Delta d)$ (i.e., some $\mathbf{x}_{0}$ in $S_{k j}(\Delta d)$ ), we have

$$
\begin{aligned}
& \mathbb{P}\left(\mathbf{0} \in \mathbf{T}_{k j}^{(2)}(\Delta d) \mid \mathcal{R}=R_{0}, \text { tier }=k\right) \\
= & 1-\exp \left(-\lambda_{j}\left|S_{k j}(\Delta d)\right|\right) \\
= & 1-\exp \left(-2 \lambda_{j} \Delta d R_{0} \mathcal{F}\left(\beta_{k j}\right)+\mathcal{O}\left(\Delta d^{2}\right)\right) \\
= & 2 \lambda_{j} \Delta d R_{0} \mathcal{F}\left(\beta_{k j}\right)+\mathcal{O}\left(\Delta d^{2}\right),
\end{aligned}
$$

which completes the proof of Case 1.

Case 2: $\beta_{k j}<1$. The proof is similar to that of Case 1 .

Case 3: $\beta_{k j}=1$. The proof of this case is relatively simple, which is omitted due to space limitation.

\subsection{Proof of Theorem 2}

Proof. Similar to the proof of Theorem 1, $\mathbf{0} \in \mathbf{T}_{k j}^{(2)}(\Delta d)$ is equivalent to the event that there is at least one point of $\Phi_{j}$ in the region $S_{k j}(\Delta d)$, given that no point of $\Phi_{j}$ is in

${ }^{1}$ If $k=j$, it is the reduced Palm point process [20] corresponding to all tier- $k$ BSs other than the reference BS. 
$\mathcal{B}\left(\mathbf{0}, R_{0} / \beta_{k j}\right)$. Note that $S_{k j}(\Delta d)$ here is still expressed in (43) and shown in Fig. 8.

As labeled in Fig. 8, we also define $\Delta D_{k j}(\theta)$ as the thickness of $S_{k j}(\Delta d)$ at angular coordinate $\theta$. It can be shown that

$$
\begin{aligned}
& \Delta D_{k j}(\theta) \\
= & \sqrt{\frac{R_{0}^{2}}{\beta_{k j}^{2}}+\frac{2 \Delta d R_{0}}{\beta_{k j}^{2}} \sqrt{\left(\beta_{k j}^{2}+1\right)-2 \beta_{k j} \cos (\theta)}+\mathcal{O}\left(\Delta d^{2}\right)}-\frac{R_{0}}{\beta_{k j}} \\
= & \Delta d \frac{\sqrt{\beta_{k j}^{2}+1-2 \beta_{k j} \cos (\theta)}}{\beta_{k j}}+\mathcal{O}\left(\Delta d^{2}\right) .
\end{aligned}
$$

Then, we have

$$
\begin{aligned}
& \mathbb{P}\left(\mathbf{0} \in \mathbf{T}_{k j}^{(2)}(\Delta d) \mid \mathcal{R}=R_{0}, \text { tier }=k\right) \\
= & \mathbb{P}\left(\Phi_{j} \bigcap S_{k j}(\Delta d) \neq \emptyset \mid \Phi_{j} \bigcap \mathcal{B}\left(\mathbf{0}, R_{0} / \beta_{k j}\right)=\emptyset\right) \\
= & \frac{1}{\pi} \int_{0}^{\pi} \frac{\operatorname{ccdf}_{\mathcal{R}_{j}}\left(\frac{R_{0}}{\beta_{k j}}\right)-\operatorname{ccdf}_{\mathcal{R}_{j}}\left(\frac{R_{0}}{\beta_{k j}}+\Delta D_{k j}(\theta)\right)+\mathcal{O}\left(\Delta d^{2}\right)}{\operatorname{ccdf}_{\mathcal{R}_{j}}\left(R_{0} / \beta_{k j}\right)} \mathrm{d} \theta \\
= & \frac{1}{\pi} \int_{0}^{\pi} \Delta D_{k j}(\theta) \mathrm{d} \theta \frac{\operatorname{pdf}_{\mathcal{R}_{j}}\left(R_{0} / \beta_{k j}\right)}{\operatorname{ccdf}_{\mathcal{R}_{j}}\left(R_{0} / \beta_{k j}\right)}+\mathcal{O}\left(\Delta d^{2}\right) \\
= & \frac{1}{\pi} \int_{0}^{\pi} \Delta d \frac{\sqrt{\beta_{k j}^{2}+1-2 \beta_{k j} \cos (\theta)}}{\beta_{k j}} \mathrm{~d} \theta \frac{\operatorname{pdf}_{\mathcal{R}_{j}}\left(R_{0} / \beta_{k j}\right)}{\operatorname{ccdf}_{\mathcal{R}_{j}}\left(R_{0} / \beta_{k j}\right)}+\mathcal{O}\left(\Delta d^{2}\right) \\
= & \frac{1}{\pi} \mathcal{F}\left(\beta_{k j}\right) \beta_{k j} \Delta d \frac{\operatorname{pdf}_{\mathcal{R}_{j}}\left(R_{0} \beta_{j k}\right)}{\operatorname{ccdf}_{\mathcal{R}_{j}}\left(R_{0} \beta_{j k}\right)}+\mathcal{O}\left(\Delta d^{2}\right),
\end{aligned}
$$

which completes the proof.

\section{REFERENCES}

[1] J. G. Andrews, F. Baccelli, and R. Ganti. A tractable approach to coverage and rate in cellular networks. IEEE Trans. on Communications, 59(11):3122-3134, Nov. 2011.

[2] A. Anpalagan and I. Katzela. Overlaid cellular system design, with cell selection criteria for mobile wireless users. In Proc. of IEEE Canadian Conference on Electrical and Computer Engineering, Edmonton, Canada, May 1999.

[3] F. Ashtiani, J. Salehi, and M. Aref. Mobility modeling and analytical solution for spatial traffic distribution in wireless multimedia networks. IEEE Journal on Selected Areas in Communications, 21(10):1699 - 1709, Dec. 2003.

[4] F. Baccelli and B. Blaszczyszyn. Stochastic geometry and wireless networks, volume 1: Theory. Foundations and Trends in Networking, 3(3-4):249 - 449, 2009.

[5] W. Bao and B. Liang. Insensitivity of user distribution in multicell networks under general mobility and session patterns. IEEE Trans. on Wireless Communications, 12(12):6244-6254, Dec. 2013.

[6] W. Bao and B. Liang. Handoff rate analysis in heterogeneous cellular networks: a stochastic geometric approach. In Proc. of ACM MSWiM, Montreal, Canada, 2014.

[7] W. Bao and B. Liang. Structured spectrum allocation and user association in heterogeneous cellular networks. In Proc. of IEEE INFOCOM, Toronto, Canada, Apr. 2014.

[8] W. Bao and B. Liang. Radio resource allocation in heterogeneous wireless networks: a spatial-temporal perspective. In Proc. of IEEE INFOCOM, Hong Kong, China, Apr. 2015.

[9] W. Bao and B. Liang. Stochastic geometric analysis of user mobility in heterogeneous wireless networks. IEEE Journal on Selected Areas in Communications, Special Issue on Recent Advances in Heterogeneous Cellular Networks, 2015.

[10] Y. Chen, J. Kurose, and D. Towsley. A mixed queueing network model of mobility in a campus wireless network. In Proc. of IEEE INFOCOM, Orlando, FL, Mar. 2012.

[11] W. C. Cheung, T. Q. S. Quek, and M. Kountouris. Throughput optimization, spectrum allocation, and access control in two-tier femtocell networks. IEEE Journal on Selected Areas in Communications, 30(3):561-574, Apr. 2012.
[12] H. Dhillon, R. Ganti, F. Baccelli, and J. G. Andrews. Modeling and analysis of K-tier downlink heterogeneous cellular networks. IEEE Journal on Selected Areas in Communications, 30(3):550-560, Apr. 2012.

[13] A. Farbod and B. Liang. Structured admission control policies in heterogeneous wireless networks with mesh underlay. In Proc. of IEEE INFOCOM, Rio de Janeiro, Brazil, Apr. 2009.

[14] H. Federer. Geometric Measure Theory. Springer, 1969.

[15] A. Ghosh, R. Jana, V. Ramaswami, J. Rowland, and N. Shankaranarayanan. Modeling and characterization of large-scale Wi-Fi traffic in public hot-spots. In Proc. of IEEE INFOCOM, Shanghai, China, Apr. 2011.

[16] K. Gulati, B. Evans, J. Andrews, and K. Tinsley. Statistics of co-channel interference in a field of poisson and poisson-poisson clustered interferers. IEEE Trans. on Signal Processing, 58(12):6207-6222, Dec. 2010.

[17] A. Hasib and A. Fapojuwo. Mobility model for heterogeneous wireless networks and its application in common radio resource management. IET Communications, 2(9):1186-1195, Oct. 2008.

[18] J. Hou and D. O'brien. Vertical handover-decision-making algorithm using fuzzy logic for the integrated radio-and-OW system. IEEE Trans. on Wireless Communications, 5(1):176-185, Jan. 2006.

[19] Z. Jako and G. Jeney. Outage probability in poisson-cluster-based lte two-tier femtocell networks. Wiley Wireless Communications and Mobile Computing, Jun. 2014.

[20] H.-S. Jo, Y. J. Sang, P. Xia, and J. G. Andrews. Heterogeneous cellular networks with flexible cell association: A comprehensive downlink SINR analysis. IEEE Trans. on Wireless Communications, 11(10):3484-3495, Oct. 2012.

[21] Y. Kirsal, E. Gemikonakli, E. Ever, G. Mapp, and O. Gemikonakli. An analytical approach for performance analysis of handoffs in the next generation integrated cellular networks and WLANs. In Proc. of International Conference on Computer Communications and Networks, Zurich, Switzerland, Aug. 2010.

[22] C. W. Lee, L. M. Chen, M. C. Chen, and Y. S. Sun. A framework of handoffs in wireless overlay networks based on mobile IPv6. IEEE Journal on Selected Areas in Communications, 23(11):2118 - 2128, Nov. 2005.

[23] X. Lin, R. Ganti, P. Fleming, and J. Andrews. Towards understanding the fundamentals of mobility in cellular networks. IEEE Trans. on Wireless Communications, 12(4):1686-1698, Apr. 2013.

[24] Y. Lin, W. Bao, W. Yu, and B. Liang. Optimizing user association and spectrum allocation in HetNets: a utility perspective. IEEE Journal on Selected Areas in Communications, Special Issue on Recent Advances in Heterogeneous Cellular Networks, 2015.

[25] M. Liu, Z. Li, X. Guo, and E. Dutkiewicz. Performance analysis and optimization of handoff algorithms in heterogeneous wireless networks. IEEE Trans. on Mobile Computing, 7(7):846-857, Jul. 2008.

[26] G. Pollini. Trends in handover design. IEEE Communications Magazine, 34(3):82-90, Mar. 1996.

[27] T. S. Rappaport. Wireless Communications: Principles and Practice. Prentice Hall, second edition, 2002.

[28] N. Shenoy and B. Hartpence. A mobility model for cost analysis in integrated cellular/WLANs. In Proc. of International Conference on Computer Communications and Networks, Chicago, IL, Oct. 2004.

[29] S. Singh, H. Dhillon, and J. G. Andrews. Offloading in heterogeneous networks: Modeling, analysis, and design insights. IEEE Trans. on Wireless Communications, 12(5):2484-2497, May 2013.

[30] M. Stemm and R. H. Katz. Vertical handoffs in wireless overlay networks. ACM/Springer Mobile Networks and Applications, 3(4):335-350, Jan. 1998.

[31] E. Stevens-Navarro, Y. Lin, and V. Wong. An MDP-based vertical handoff decision algorithm for heterogeneous wireless networks. IEEE Trans. on Vehicular Technology, 57(2):1243-1254, Mar. 2008.

[32] D. Stoyan, W. Kendall, and J. Mecke. Stochastic Geometry and Its Applications. Wiley, second edition, 1995.

[33] Y. Zhong and W. Zhang. Multi-channel hybrid access femtocells: A stochastic geometric analysis. IEEE Trans. on Communications, 61(7):3016-3026, Jul. 2013. 